\title{
Synthesis, Growth, and Characterization of Bisglycine Hydrobromide Single Crystal
}

\author{
Koteeswari Pandurangan ${ }^{1}$ and Sagadevan Suresh ${ }^{2}$ \\ ${ }^{1}$ Department of Physics, Madha Engineering College, Kundrathur, Chennai, India \\ ${ }^{2}$ Department of Physics, Sree Sastha Institute of Engineering and Technology, Chembarambakkam, Chennai 600123, India \\ Correspondence should be addressed to Sagadevan Suresh; sureshsagadevan@yahoo.co.in
}

Received 27 October 2013; Revised 12 June 2014; Accepted 13 June 2014; Published 29 June 2014

Academic Editor: Rodrigo Martins

Copyright (c) 2014 K. Pandurangan and S. Suresh. This is an open access article distributed under the Creative Commons Attribution License, which permits unrestricted use, distribution, and reproduction in any medium, provided the original work is properly cited.

\begin{abstract}
Single crystals of BGHB were grown by slow evaporation technique. The unit cell dimensions and space group of the grown crystals were confirmed by single crystal X-ray diffraction. The modes of vibration of the molecules and the presence of functional groups were identified using FTIR technique. The microhardness study shows that the Vickers hardness number of the crystal increases with the increase in applied load. The optical properties of the crystals were determined using UV-Visible spectroscopy. The thermal properties of the grown crystal were also determined. The refractive index was determined as 1.396 using Brewster's angle method. The emission of green light on passing the Nd: YAG laser light confirmed the second harmonic generation property of the crystals and the SHG efficiency of the crystals was found to be higher than that of KDP. The dielectric constant and dielectric loss measurements were carried out for different temperatures and frequencies. The ac conductivity study of the crystals was also discussed. The photoconductivity studies confirm that the grown crystal has negative photoconductivity nature. The etching studies were carried out to study the formation of etch pits.
\end{abstract}

\section{Introduction}

Nonlinear optical (NLO) applications demand good quality single crystals, which inherit large NLO coefficient, coupled with improved physical parameters. One potentially attractive system, where there is a potential for realizing very large second order nonlinear coefficient is based on organic crystals. Organic materials have been of particular interest because the nonlinear optical responses in this broad class of materials is microscopic in origin, offering an opportunity to use theoretical modeling coupled with synthetic flexibility to design and produce novel materials [1]. Further investigations on organic NLO materials have subsequently produced very good materials with highly attractive characteristics. Amino acid family crystals have been subjected to extensive investigation during the recent decades for their nonlinear optical properties [2]. In the present day technological society, nonlinear optical (NLO) materials are most useful in the area of optical data storage, lasers, optical signal processing, second harmonic generation, and so forth. Even though varieties of nonlinear optical materials are available, their applications are limited due to physical and chemical properties [3]. Amino acids are interesting materials for NLO applications as they contain a proton donor carboxyl acid $(\mathrm{COOH})$ group and the proton acceptor amino $\left(\mathrm{NH}_{2}\right)$ group in them. Amino acids are widely utilized because they not only contain chiral carbon atoms directing the crystallization process in the noncentrosymmetric space group, but also possess zwitter-ionic nature favouring crystal hardness [4]. The present investigation deals with the growth of bisglycine hydrobromide (BGHB) single crystal that was grown by slow solvent evaporation technique. The grown crystals were characterized by single crystal and powder X-ray analysis, FTIR and UV spectral analysis, thermal analysis, microhardness, dielectric, SHG, and photoconductivity measurements. The results of these studies have been discussed in this paper in detail. 


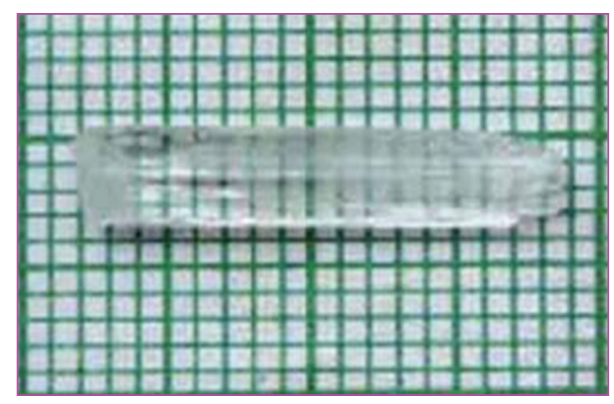

FIGURE 1: Photograph of grown BGHB single crystal.

\section{Experimental Procedure}

Bisglycine hydrobromide salt was synthesized by dissolving glycine and hydrobromic acid in stoichiometric ratio $(3: 1)$ in double distilled water. The solution was stirred continuously using a magnetic stirrer. The obtained saturated solution was further purified and allowed to evaporate at higher temperature which yields powder form of the synthesized bisglycine hydrobromide. Synthesized material was purified by repeated recrystallization process. Tiny seed crystals with good transparency were obtained due to spontaneous nucleation. Among them, a defect free seed crystal was selected and suspended in the mother solution, which was allowed to evaporate at room temperature. Large size single crystals were obtained due to the collection of monomers at the seed crystal sites from the mother solution, after the nucleation and growth processes were completed. After a period of 24 days, colourless and transparent crystals were obtained with dimensions $\left(17 \times 7 \times 6 \mathrm{~mm}^{3}\right)$. Figure 1 shows a grown crystal of BGHB.

\section{Results and Discussion}

3.1. Single-Crystal X-Ray Diffraction. Single-crystal X-ray diffraction is an analytical technique to determine the actual arrangement of atoms within a crystalline specimen. Singlecrystal X-ray diffraction (XRD) is a nondestructive tool to analyze crystal structure of compounds, which can be grown as single crystals. XRD is employed for finding unit cell parameters, space groups, and three-dimensional coordinates of atoms in the unit cell. The single-crystal X-ray diffraction analysis of the grown crystals was carried out to identify the cell parameters using an ENRAF NONIUS CAD4 automatic $\mathrm{X}$-ray diffractometer. The lattice parameters are estimated to be $a=5.38 \AA \AA, b=8.16 \AA \AA, c=18.38 \AA, \alpha=\beta=90^{\circ}$, and $\beta=111.52^{\circ}$, and hence the crystal belongs to the monoclinic crystal system which agrees well with the available reported literature values [5].

3.2. Powder X-Ray Diffraction. The powder X-ray diffraction analysis was carried out to confirm the crystallinity and also to ascertain the purity of the grown BGHB crystal. Powder $\mathrm{XRD}$ pattern was recorded by scanning the sample over the range $0-90^{\circ}$ at a scan speed of $0.02^{\circ} / \mathrm{min}$. The recorded XRD pattern of $\mathrm{BGHB}$ is shown in Figure 2 and the planes were

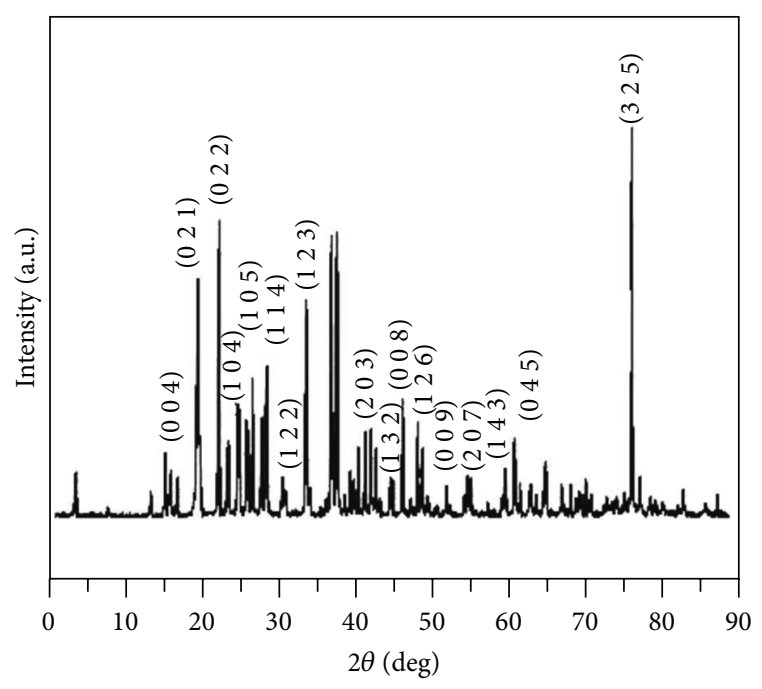

FIGURE 2: Powder X-ray diffraction pattern of BGHB single crystal.

indexed. The appearance of sharp and strong peaks confirms good crystallinity of the grown BGHB crystal. From the data, the lattice parameters were calculated as $a=5.38 \AA, b=$ $8.16 \AA, c=18.38 \AA, \alpha=\beta=90^{\circ}$, and $\gamma=111.80$. The results agree well with single-crystal XRD results and also with the available literature values [5].

3.3. FTIR Spectrum Analysis. The FTIR spectrum of bisglycine hydrobromide was recorded in the region $4000 \mathrm{~cm}^{-1}$ $-400 \mathrm{~cm}^{-1}$ employing BRUKER IFS $66 \mathrm{~V}$ spectrometer. In the FTIR spectrum shown in Figure 3, there is a broad band between 2000 and $3500 \mathrm{~cm}^{-1}$. The recorded FTIR spectrum was compared with the results of the above researchers and also with the standard spectra of the functional groups [6]. The sharp peak at 3429,3113 , and $2897 \mathrm{~cm}^{-1}$ may be assigned to $\mathrm{NH}_{3}{ }^{+}$stretching band [7]. The peaks at $2692 \mathrm{~cm}^{-1}$ and $2602 \mathrm{~cm}^{-1}$ are attributed to the $\mathrm{C}-\mathrm{H}$ stretching mode vibrations [8]. The overtone region contains a band near $2000-1800 \mathrm{~cm}^{-1}$ which is assigned to the combination of the asymmetrical $\mathrm{NH}_{3}{ }^{+}$bending and torsional oscillations of the $\mathrm{NH}_{3}{ }^{+}$groups. The intense peaks at 1742, 1714, and $1123 \mathrm{~cm}^{-1}$ indicate the $\mathrm{C}=\mathrm{O}$ stretching of the COO- group [9]. The asymmetric and symmetric stretching modes of COO- groups are confirmed by the peaks at 1615, 1496, 1445, 1332 , and $1253 \mathrm{~cm}^{-1}$ [10].

This observation confirms that one glycine is exists in zwitter-ionic form and the peak at $672 \mathrm{~cm}^{-1}$ is due to $\mathrm{N}-\mathrm{H}$ out-of-plane bending vibrations [11]. The torsional oscillation of $\mathrm{NH}_{3}{ }^{+}$is revealed by the peak at $542 \mathrm{~cm}^{-1}$. The strong carbonyl absorption at $1742 \mathrm{~cm}^{-1}$ characterizes $\alpha$-amino acid hydrobromides [12]. FTIR spectral analysis thus identifies the presence of functional group in the grown material BGHB.

3.4. UV-Vis-NIR Spectral Analysis. The optical transmission spectrum of BGHB single crystal was recorded in the wavelength region 200-2000 $\mathrm{nm}$ and it is shown in Figure 4. For 


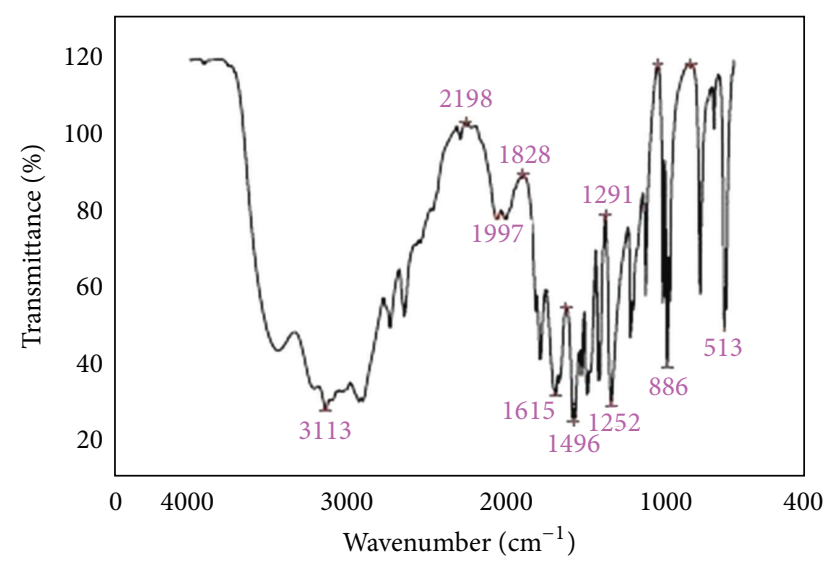

FIGURE 3: FTIR spectrum of BGHB single crystal.

optical fabrications, the crystal should be highly transparent in the considered region of wavelength $[13,14]$. The favorable transmittance of the crystal in the entire visible region suggests its suitability for second harmonic generation [15]. The UV absorption edge for the grown crystal was observed to be around $230 \mathrm{~nm}$. The lower cut-off wavelength is found to be $230 \mathrm{~nm}$ for BGHB which is in fairly good agreement with reported value [5]. The dependence of optical absorption coefficient on photon energy helps to study the band structure and type of transition of electrons [16]. The optical absorption coefficient $(\alpha)$ was calculated from transmittance using the following relation:

$$
\alpha=\frac{1}{d} \log \left(\frac{1}{T}\right)
$$

where $T$ is the transmittance and $d$ is the thickness of the crystal. As a direct band gap material, the crystal under study has an absorption coefficient $(\alpha)$ obeying the following relation for high photon energies $(h v)$ :

$$
\alpha=\frac{A\left(h v-E_{g}\right)^{1 / 2}}{h v},
$$

where $E_{g}$ is the optical band gap of the crystal and $A$ is a constant. A plot of variation of $(\alpha h \nu)^{2}$ versus $h v$ is shown in Figure 5. $E_{g}$ is evaluated using the extrapolation of the linear part [17]. The energy absorption gap is of direct type and the band gap energy is found to be $3.70 \mathrm{eV}$ and the large band gap clearly indicates the wide transparency of the crystal.

From the transmission spectrum, the lower cut-off wavelength is found to be $230 \mathrm{~nm}$ and the lower percentage absorption indicates that the crystal readily allows the transmission of the laser beam in the range between $230 \mathrm{~nm}$ and $2000 \mathrm{~nm}$. It shows that the grown crystal has a good transparency in UV, visible, and near IR region indicating that it can be used for NLO applications. Using Tauc's plot, the energy gap $\left(E_{g}\right)$ was calculated as $3.70 \mathrm{eV}$. This high band gap value indicates that the grown crystal possesses dielectric behaviour to induce polarization when powerful radiation is incident on the material. The band gap for the grown crystal

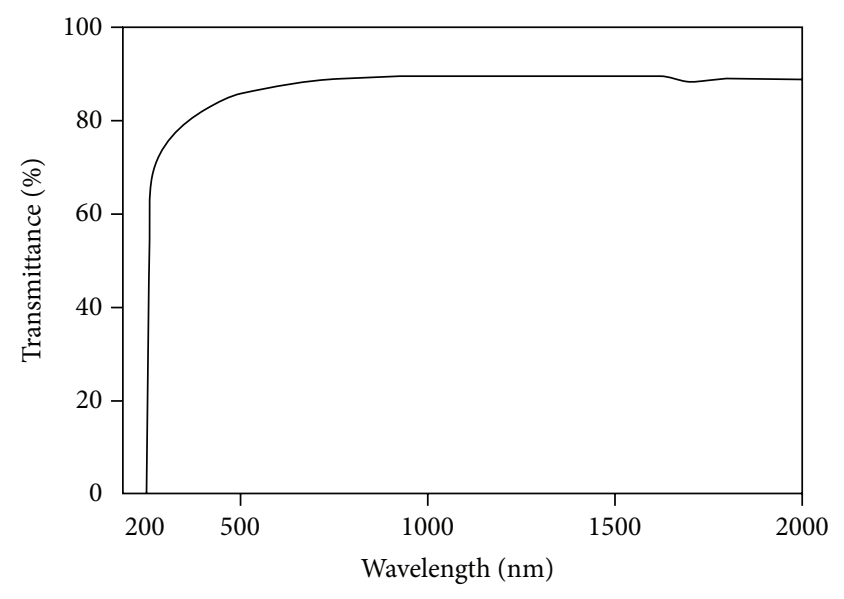

Figure 4: UV-Vis transmission spectrum of BGHB.

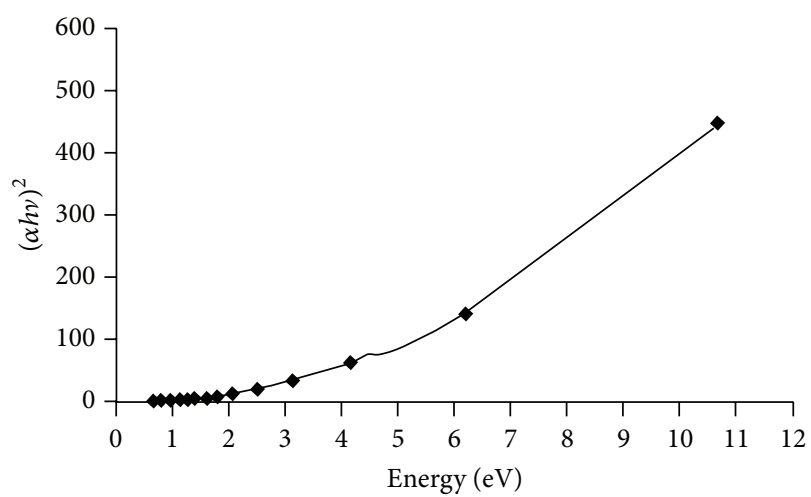

FIGURE 5: Plot of $(\alpha h \nu)^{2}$ versus photon energy for the single-crystal BGHB.

is found to be $3.70 \mathrm{eV}$, which is in good agreement with the reported value of $3.1 \mathrm{eV}$ [18]. This value confirms the dielectric behaviour of the material.

3.5. Refractive Index Measurements. Brewster's angle method was used to determine the refractive index of the crystal. A He-Ne laser of wavelength $632.8 \mathrm{~nm}$ was used as the source. The polished crystal was kept on the rotating mount at an angle varying from 0 to 90 degrees. The angular reading, when the crystal was perfectly perpendicular to beam, was noted. The crystal was rotated along the beam for maximum power output. Brewster's angle $(\theta p)$ was measured as 54.6 degrees. The refractive index of the BGHB was calculated and found to be 1.396 .

3.6. NLO Test: Kurtz Powder SHG Method. The most widely used technique for confirming the SHG efficiency of NLO materials to identify the materials with noncentrosymmetric crystal structures is the Kurtz Powder technique [19]. In this technique the powdered sample with an average particle sizes range of $125-150 \mu \mathrm{m}$ is filled in a microcapillary tube about $1.5 \mathrm{~mm}$ in diameter. Q-switched Nd: YAG laser emitting a fundamental wavelength of $1064 \mathrm{~nm}$ was used. The input laser 


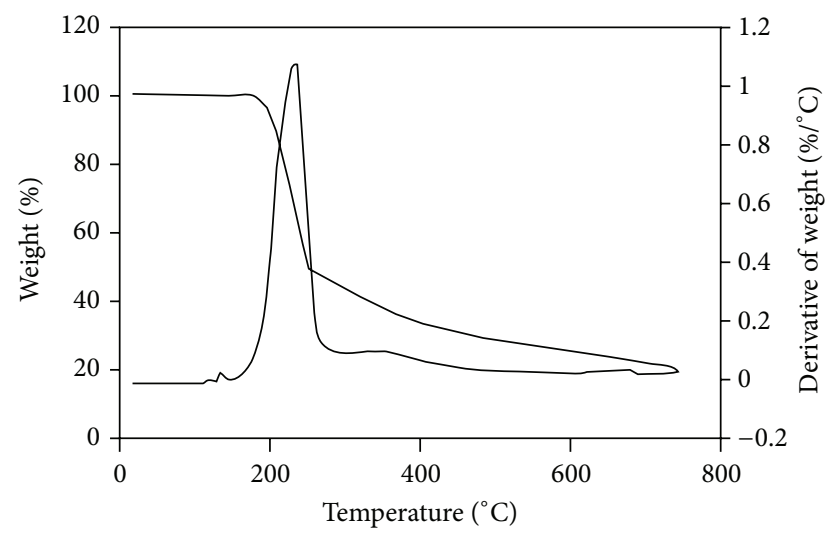

Figure 6: 6 TG/DTA of BGHB.

energy incident on the sample was $6.2 \mathrm{~mJ} /$ pulse and a pulse width of $8 \mathrm{~ns}$ with a repetition rate of $10 \mathrm{~Hz}$ was made to fall normally on the sample. The standard NLO inorganic potassium dihydrogen phosphate (KDP) was used as the reference material. The emission of green light $(\lambda=532 \mathrm{~nm})$ from the BGHB crystals confirmed their noncentrosymmetric crystal structure. The second harmonic radiation generated by the randomly oriented microcrystals was focused by a lens and detected and a photomultiplier tube. The generation of the second harmonic radiation was confirmed by a strong bright green emission emerging from the powdered sample. A potassium dihydrogen phosphate crystal was used as a reference material in the SHG measurement. The relative conversion efficiency was calculated from the output power of BGHB crystals with reference to KDP crystals. When KDP crystal was used as a reference material, it produced $62 \mathrm{mV}$ as output beam voltage. But the grown sample produced about $93 \mathrm{mV}$ as output beam voltage. Hence, it is confirmed that the material has NLO efficiency of about 1.5 times that of the KDP crystal. The nonlinear optical study confirms the SHG property of the material equal to 1.5 times that of the standard $\mathrm{KDP}$ and this prediction is comparable with the reported value of 1.25 [20].

3.7. Thermal Studies. The thermogravimetric analysis (TGA) was carried out by using TAQ-500 analyzer at a heating rate of $25^{\circ} \mathrm{C} / \mathrm{min}$. for temperature range $25-800^{\circ} \mathrm{C}$ as shown in Figure 6. The spectrum shows that there is small weight loss around $180^{\circ} \mathrm{C}$. The material starts decomposing near temperature of $248^{\circ} \mathrm{C}$, which is melting point of the $\mathrm{BGHB}$ crystal. The weight loss of $73.25 \%$ of $7.432 \mathrm{mg}$ of sample was observed in the temperature range from $230^{\circ} \mathrm{C}$ to $305.36^{\circ} \mathrm{C}$. Thereafter residue remains up to of $800^{\circ} \mathrm{C}$. It is clear that the crystal is thermally stable up to $230^{\circ} \mathrm{C}$ [21]. Hence it can be utilized for device applications till $230^{\circ} \mathrm{C}$. From the thermal analysis it is concluded that the grown crystal decomposes without melting at about $230^{\circ} \mathrm{C}$ and is stable till that temperature, which agrees with the reported value $206^{\circ} \mathrm{C}[5]$.

3.8. Microhardness Studies. The microhardness measurements were carried out with a load range from 25 to $100 \mathrm{~g}$

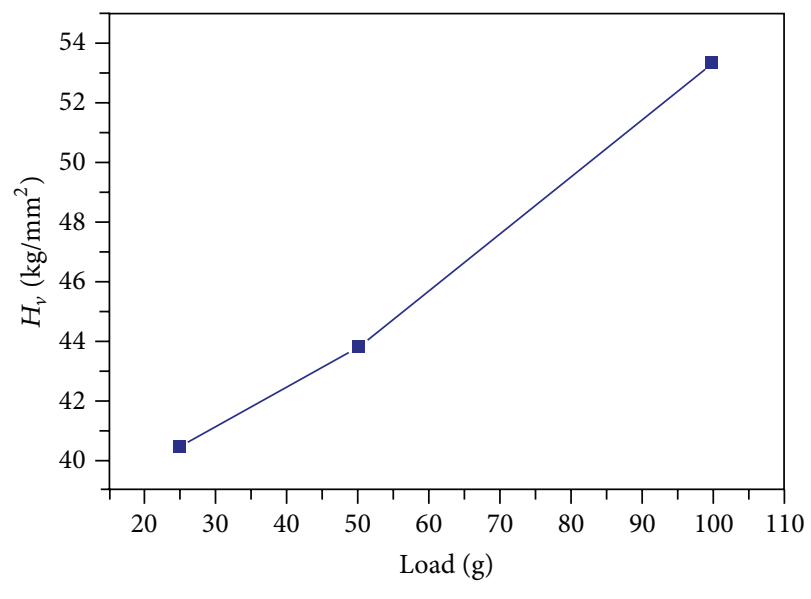

Figure 7: Variation of $H_{v}$ with load $P$.

using Vickers hardness tester (LEITZ WETZLER) fitted with a diamond pyramidal indenter and attached to an incident light microscope. The Vickers microhardness number was calculated using the relation

$$
H_{v}=1.8544\left(\frac{p}{d^{2}}\right) \mathrm{kg} / \mathrm{mm}^{2},
$$

where $p$ is the indenter load and $d$ is the diagonal length of the impression. The Figure 7 shows the variation of $P$ with Vickers hardness number $\left(H_{v}\right)$ for grown single crystal. It is evident from the plot that Vickers microhardness number increases with increasing applied load. According to Meyer's law, the relation connecting the applied load is given by

$$
\begin{gathered}
p=k_{1} d^{n}, \\
\log p=\log k+n \log d,
\end{gathered}
$$

where $n$ is the Meyer index or work hardening exponent and $k_{1}$, is the constant for a given material. The above relation indicates that " $H_{v}$ " should increase with load $p$ if $n>2$ and decrease with load $p$ when $n<2$. We have determined " $n$ " from slope of the plot that is shown in Figure 8. The value of " $n$ " for BGHB was found to be 3.15. Low value of work hardening coefficient " $n$ " illustrates fewer defects in the grown crystal. The large value of $n$ indicates large effect of dislocations. According to Onitsch [22], $n$ should lie between 1 and 1.6 for harder materials and above 1.6 for softer materials. From the hardness study, the grown BGHB crystal is found to be relatively soft material.

3.9. Dielectric Studies. The dielectric studies of the grown crystal were carried out by using the instrument, HIOCKI 3532-50 LCR HITESTER. A sample of dimension $1 \times 0.5$ $\times 0.1 \mathrm{~cm}^{3}$ having silver coating on the opposite faces was placed between the two copper electrodes and a parallel plate capacitor was thus formed. The capacitance of the sample was measured by varying the frequency from $100 \mathrm{~Hz}$ to $5 \mathrm{MHz}$ and the dielectric constant versus logarithmic frequency is plotted (Figure 9). The increase in dielectric constant at low frequency is attributed to the space charge polarization [23]. 


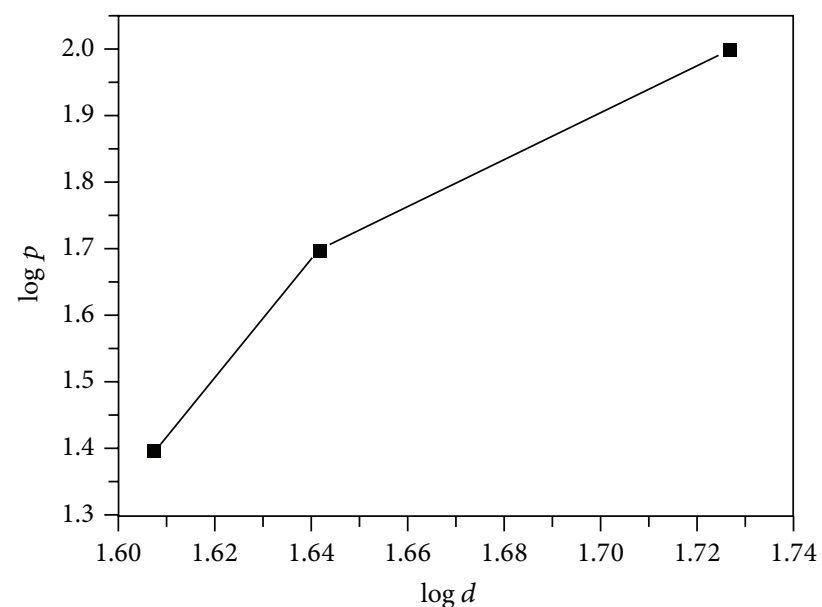

FIgure 8: Plot of $\log d$ versus $\log p$.

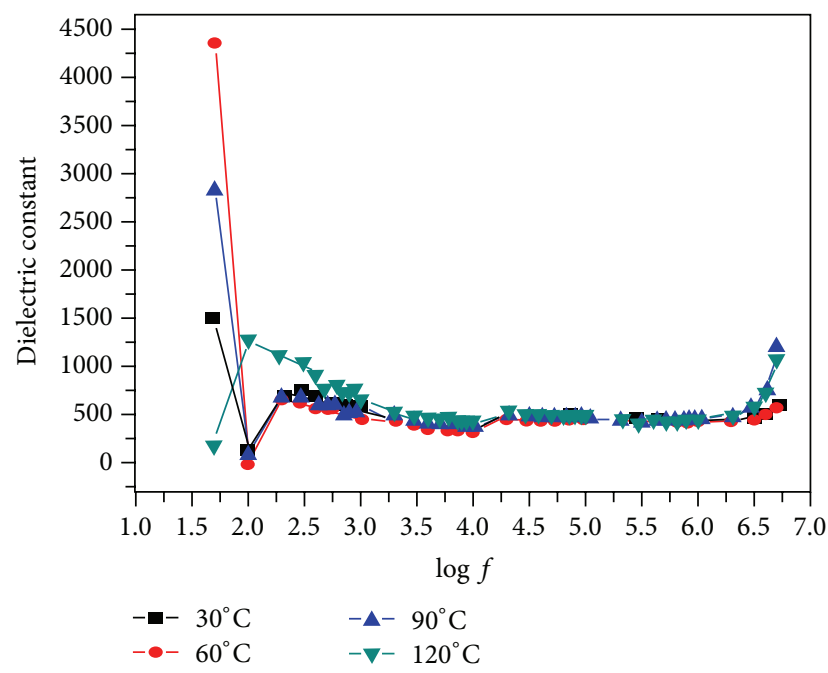

FIgURE 9: Variation of the dielectric constant against $\log f$.

The dielectric loss is also studied as a function of frequency at different temperatures (Figure 10). These curves suggest that the dielectric loss strongly depends on the frequency of the applied field, similar to that of dielectric constant which is common for the ionic system of crystals [24, 25].

3.10. AC Conductivity Study. The ac conductivity study of the BGHB crystal was carried out. In the high temperature (Intrinsic) region, the effect of impurity on electrical conduction has not made any appreciable change whereas in the low temperature (extrinsic) region, the presence of impurity in the crystal has an impact and particularly increases its conductivity. The electrical conduction in dielectrics is mainly a defect controlled process in the low temperature region (Figure 11). The presence of impurities and vacancies predominantly determines this region. The energy needed to form the defect is much larger than the energy need for its drift. It is found from the Arrhenius plot (Figure 12) for

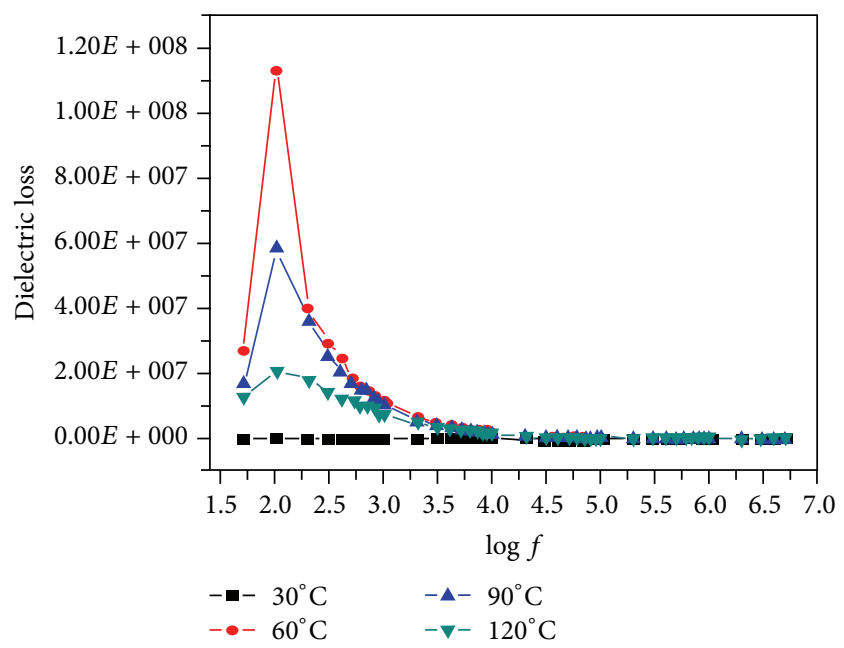

Figure 10: Variation of the dielectric loss with $\log f$.

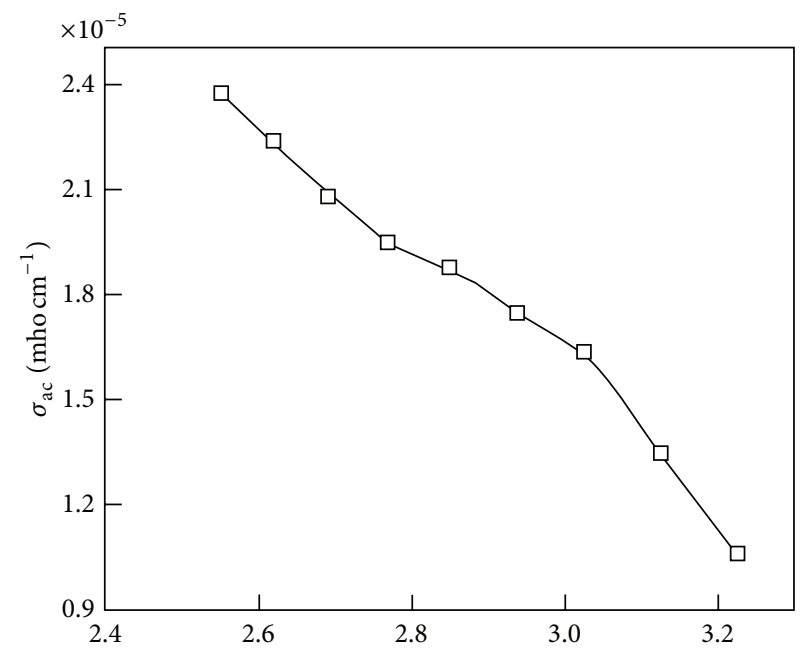

FIGURE 11: Variation of ac conductivity with $1000 / T$ for BGHB single crystal.

the BGHB crystal that the conductivity increases with temperature and the value of activation energy is found to be $0.125 \mathrm{eV}$. The conduction mechanism can be explained by the rotation of ions when the temperature of the material is increased. The rotation of ions gives local displacement of electrons in the direction of the applied field, which in turn gives rise to induced polarization when the material is subjected to high intense laser beam. The lower value of activation energy $(0.120 \mathrm{eV})$ predicted in the present investigation suggests that the crystal is free from defects. When the crystal is free from defects, the hydrogen bond interactions will not be weakened, as reported for other general amino acid groups of materials [26].

3.11. Photoconductivity Studies. The photoconductivity measurements were carried out by using Keithley 485 picoammeter. The dark current was recorded by keeping the sample unexposed to any radiation. Figure 13 shows the variation 


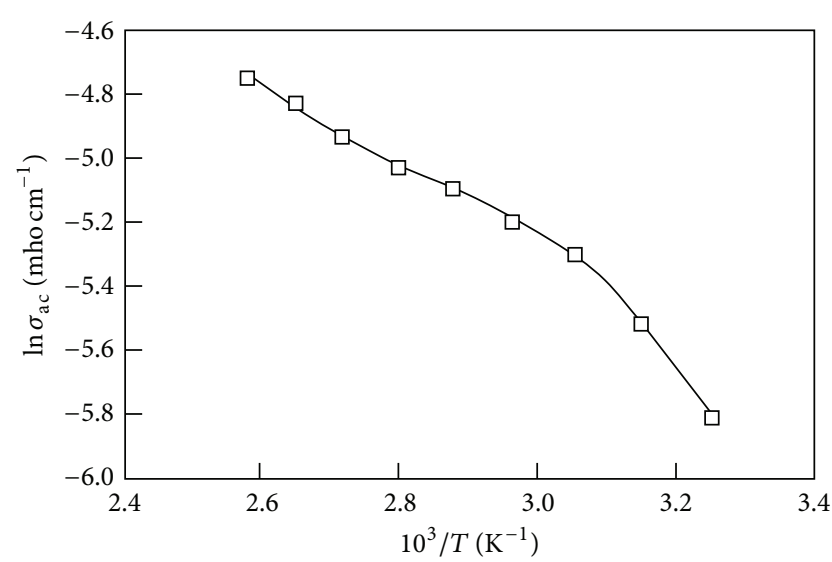

Figure 12: Plot of $\ln \left(\sigma_{\mathrm{ac}}\right)$ versus $1000 / T$ for BGHB single crystal.

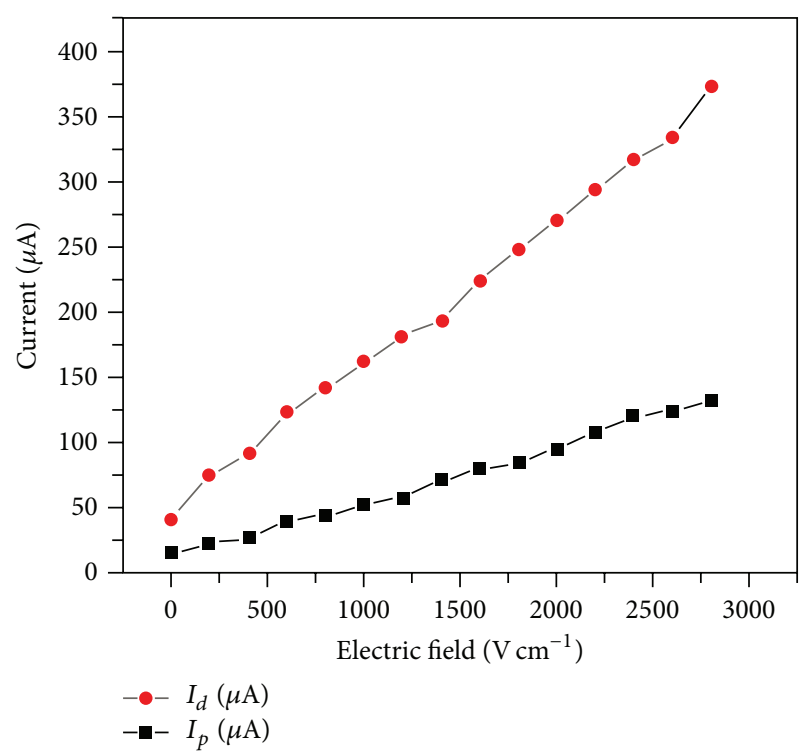

FIGURE 13: Field dependent photoconductivity of grown single crystal.

of both dark current $\left(I_{d}\right)$ and photocurrent $\left(I_{p}\right)$ with the applied electrical field. It is seen to be noted from the plots that both $I_{d}$ and $I_{p}$ of the sample increase linearly with the applied electrical field. It is observed from the plot that the dark current is always greater than the photocurrent, thus confirming the negative photoconductivity.

3.12. Etching Studies. The crystals with defects may destroy the mechanical and electrical properties, which affect the usefulness of the crystals. The nonlinear optical properties such as SHG efficiency, damage threshold, depend on the crystalline perfection. Etching is one of the selective tools to identify the defects in the grown crystals. The (100) plane of the BGHB crystal has been completely immersed for $60 \mathrm{~s}$ in the water etchant and the sample was wiped out with dry filter paper. From Figure 14, a number of etch pits have been identified with identical shape. By increasing the etching time, the pattern remains the same, but the size of the etch

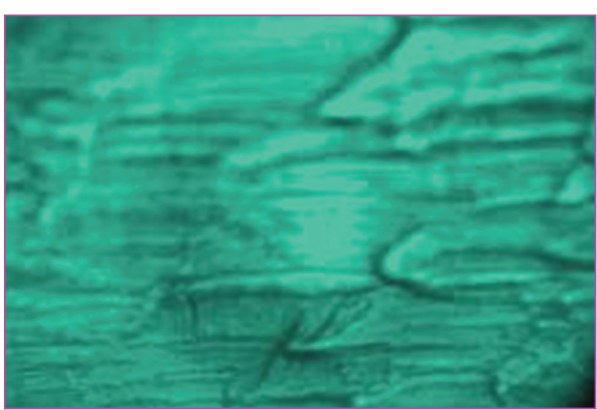

FIGURE 14: Etching photograph of BGHB.

pits has increased. The etch pit can be attributed to the initial dislocations formed at low angle boundaries or segregated impurities [27] which is strongly correlated to the formation of inclusions in the crystals which can originate from growth sector boundaries [28]. Also due to the liquid inclusions there have been drastic changes in growth condition [29]. In general, it is observed that the less number of dislocations shows the quality of the crystal.

\section{Conclusion}

A single crystal of bisglycine hydrobromide (BGHB) was grown by slow evaporation technique. The unit cell dimensions of the grown crystals were confirmed by single-crystal $\mathrm{X}$-ray diffraction. The functional groups present in the material have been confirmed by FTIR analysis. UV-Visible absorption spectrum shows excellent transmission in the entire visible region. The band gap energy for the grown crystal is found to be $3.70 \mathrm{eV}$. NLO study reveals that the grown crystal has SHG efficiency equal to that of KDP crystal. From thermal analysis, it is found that the crystal can retain its stability up to $230^{\circ} \mathrm{C}$. The mechanical studies were carried out for the grown crystal. The microhardness studies indicate that the Vickers hardness number of the crystal increases with the increase in applied load. The dielectric constant and dielectric loss measurements were carried out at different temperatures and frequencies. The activation energy was determined from the plot of ac conductivity. The photoconductivity studies confirm that this material has negative photoconductivity nature. The etching studies were carried to study the formation of etch pits in water solvent. This material exhibits NLO behaviour remarkably due to its better optical and dielectric properties.

\section{Conflict of Interests}

The authors declare that there is no conflict of interests regarding the publication of this paper.

\section{References}

[1] B. N. Moolya and S. M. Dharmapraksh, "Growth and characterization of nonlinear optical diglycinehydrobromide single crystals," Materials Letters, vol. 61, no. 17, pp. 3559-3562, 2007. 
[2] K. Ambujam, S. Selvakumar, D. P. Anand, G. Mohamed, and P. Sagayaraj, "Crystal growth, optical, mechanical and electrical properties of organic NLO material $\gamma$-glycine," Crystal Research and Technology, vol. 41, no. 7, pp. 671-677, 2006.

[3] M. D. Aggarwal, J. Stephens, A. K. Batra, and R. B. Lal, "Bulk crystal growth and characterization of semiorganic nonlinear optical materials," Journal of Optoelectronics and Advanced Materials, vol. 5, no. 3, pp. 555-562, 2003.

[4] M. N. Bhat and S. M. Dharmaprakash, "Growth of nonlinear optical $\gamma$-glycine crystals," Journal of Crystal Growth, vol. 236, no. 1-3, pp. 376-380, 2002.

[5] S. S. Hussaini, N. R. Dhumane, and D. Mahendra Shirsat, "Growth and characterizations of bis glycine hydrogen bromide (BGHB) single crystal: new nonlinear optical material," Recent Research in Science and Technology, vol. 4, pp. 10-12, 2012.

[6] T. Pal and T. Kar, "Optical, mechanical and thermal studies of nonlinear optical crystal L-arginine acetate," Materials Chemistry and Physics, vol. 91, pp. 343-347, 2005.

[7] N. Vijayan, S. Rajasekaran, G. Bhagavannarayana et al., "Growth and characterization of nonlinear optical amino acid single crystal: L-alanine," Crystal Growth \& Design, vol. 6, no. 11, pp. 2441-2445, 2006.

[8] R. M. Silverstein, G. C. Bassler, and T. C. Morrill, Spectrometric Identification of Organic Compounds, John Wiley \& Sons, New York, NY, USA, 1981.

[9] R. M. Silverstein and F. X. Webster, Spectroscopic Identification of Organic Compounds, Wiley, New York, NY, USA, 6th edition, 1998.

[10] G. Herzberg, Infrared and Raman Spectra of Polyatomic Molecules, Van Nostran Reenhold, New York, NY, USA, 1945.

[11] D. Jayalakshmi and J. Kumar, "Growth and characterization of Bis Thiourea Zinc Acetate (BTZA)," Crystal Research and Technology, vol. 41, no. 1, pp. 37-40, 2006.

[12] V. Krishnakumar, S. Sivakumar, R. Nagalakshmi, S. Bhuvaneswari, and M. Rajaboopathi, "Effect of doping an organic molecule ligand on TGS single crystals," Spectrochimica Acta A, vol. 71, no. 2, pp. 480-485, 2008.

[13] V. Krishnakumar, R. Nagalakshmi, and P. Janaki, "Growth and spectroscopic characterization of a new organic nonlinear optical crystal-8-hydroxyquinoline," Spectrochimica Acta A, vol. 61, no. 6, pp. 1097-1103, 2005.

[14] V. Krishnakumar and R. John Xavier, "FT Raman and FTIR spectral studies of 3-mercapto-1,2,4-triazole," Spectrochimica Acta A: Molecular and Biomolecular Spectroscopy, vol. 60, no. 3, pp. 709-714, 2004.

[15] A. Roshan S., C. Joseph, and M. A. Ittyachen, "Growth and characterization of a new metal-organic crystal: potassium thiourea bromide," Materials Letters, vol. 49, no. 5, pp. 299-302, 2001.

[16] N. Tigau, V. Ciupina, G. Prodan, G. I. Rusu, C. Gheorghies, and E. Vasile, "Influence of thermal annealing in air on the structural and optical properties of amorphous antimony trisulfide thin films," Journal of Optoelectronics and Advanced Materials, vol. 6, no. 1, pp. 211-217, 2004.

[17] A. K. Chawla, D. Kaur, and R. Chandra, "Structural and optical characterization of $\mathrm{ZnO}$ nanocrystalline films deposited by sputtering," Optical Materials, vol. 29, no. 8, pp. 995-998, 2007.

[18] R. Surekha, P. Sagayaraj, and K. Ambujam, "Third order nonlinear optical, luminescence and electrical properties of bis glycine hydrobromide single crystals," Optical Materials, vol. 36, no. 5, pp. 945-949, 2014.
[19] S. K. Kurtz and T. T. Perry, "A powder technique for the evaluation of nonlinear optical materials," Journal of Applied Physics, vol. 39, no. 8, pp. 3798-3813, 1968.

[20] S. Sampthkrishnan, N. Balamurugan, R. Kumutha, Y. Vidyalakshmi, and S. Muthu, "Growth and characterization of new non linear optical Bis-Glycine Hydro Bromide (BGHB) single crystal," Journal of Minerals \& Materials Characterization \& Engineering, vol. 11, no. 6, pp. 597-607, 2012.

[21] K. Sugandhi, S. Dinakaran, M. Jose et al., "Crystalline perfection, spectroscopic investigations and transport properties of trisglycine zinc chloride NLO single crystal," Physica B: Condensed Matter, vol. 405, no. 18, pp. 3929-3935, 2010.

[22] E. M. Onitsch, "The present status of testing the hardness of materials," Microscope, vol. 95, pp. 12-14, 1950.

[23] J. C. Anderson, Dielectrics, Chapman \& Hall, New York, NY, USA, 1964.

[24] K. V. Rao and A. Smakula, "Dielectric properties of cobalt oxide, nickel oxide, and their mixed crystals," Journal of Applied Physics, vol. 36, no. 6, pp. 2031-2038, 1965.

[25] K. V. Rao and A. Smakula, "Dielectric properties of alkaline earth fluoride single crystals," Journal of Applied Physics, vol. 37, no. 1, pp. 319-323, 1966.

[26] S. Suresh and K. Anand, "Studies on optical, dielectric and electrical conductivity properties of zinc succinate NLO single crystal," Advances in Applied Science Research, vol. 3, no. 2, pp. 815-820, 2012.

[27] M. Senthil Pandian, N. Balamurugan, G. Bhagavannarayana, and P. Ramasamy, "Characterization of $\langle 010\rangle$ directed KAP single crystals grown by Sankaranarayanan-Ramasamy (SR) method," Journal of Crystal Growth, vol. 310, no. 18, pp. 41434147, 2008

[28] H. L. Bhat, "X-ray topographic assessment of dislocations in crystals grown from solution," Progress in Crystal Growth and Characterization, vol. 11, no. 2, pp. 57-87, 1985.

[29] K. K. Rao and V. Surender, "Surface studies on as-grown (111) faces of sodium bromate crystals," Bulletin of Materials Science, vol. 24 , no. 6, pp. 665-669, 2001. 

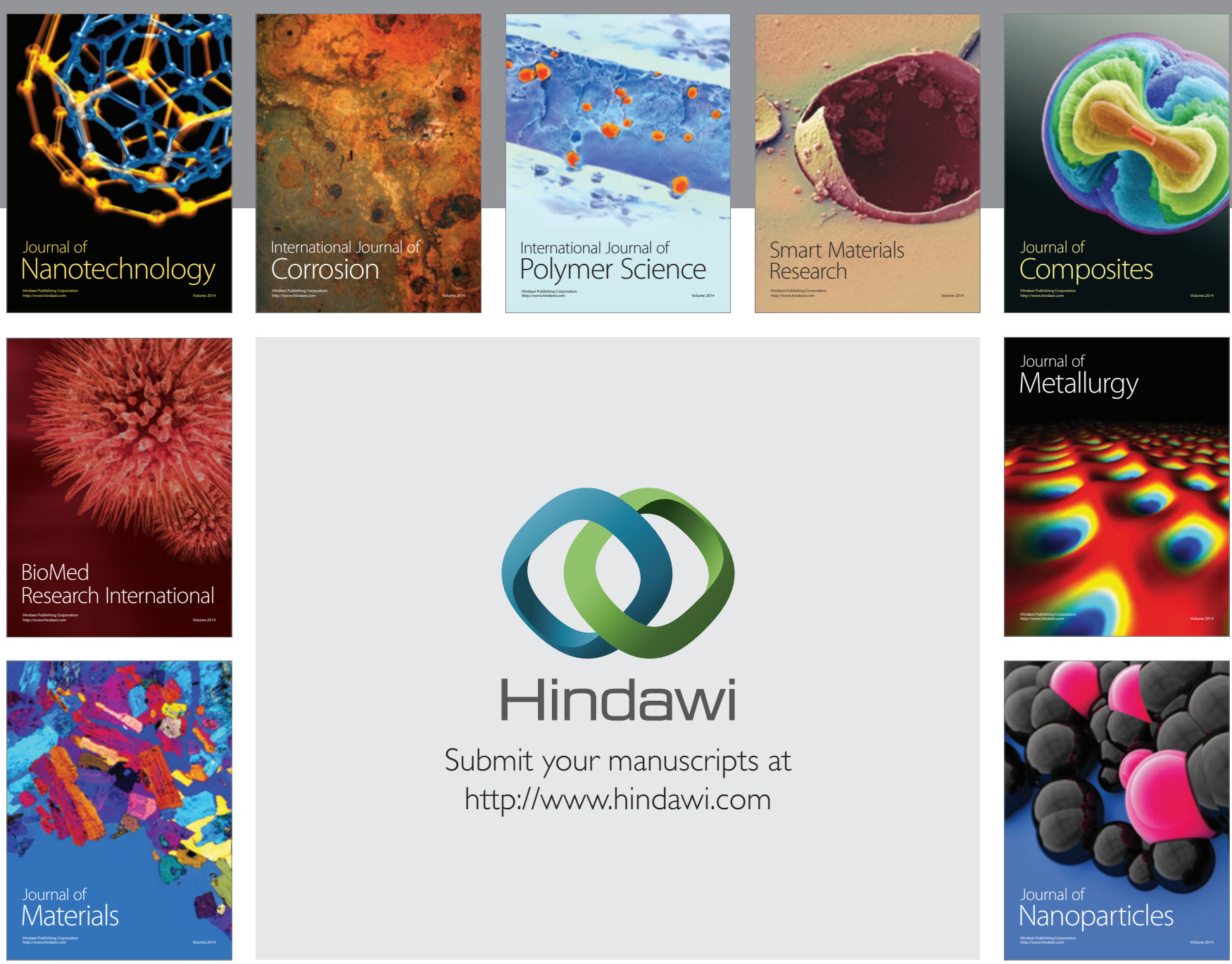

Submit your manuscripts at http://www.hindawi.com
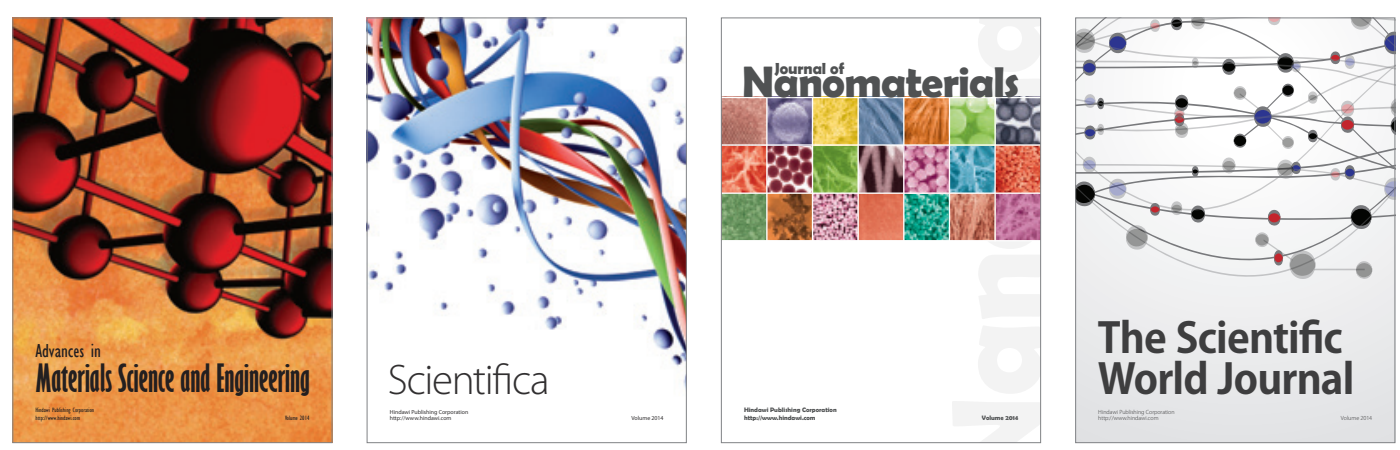

\section{The Scientific World Journal}
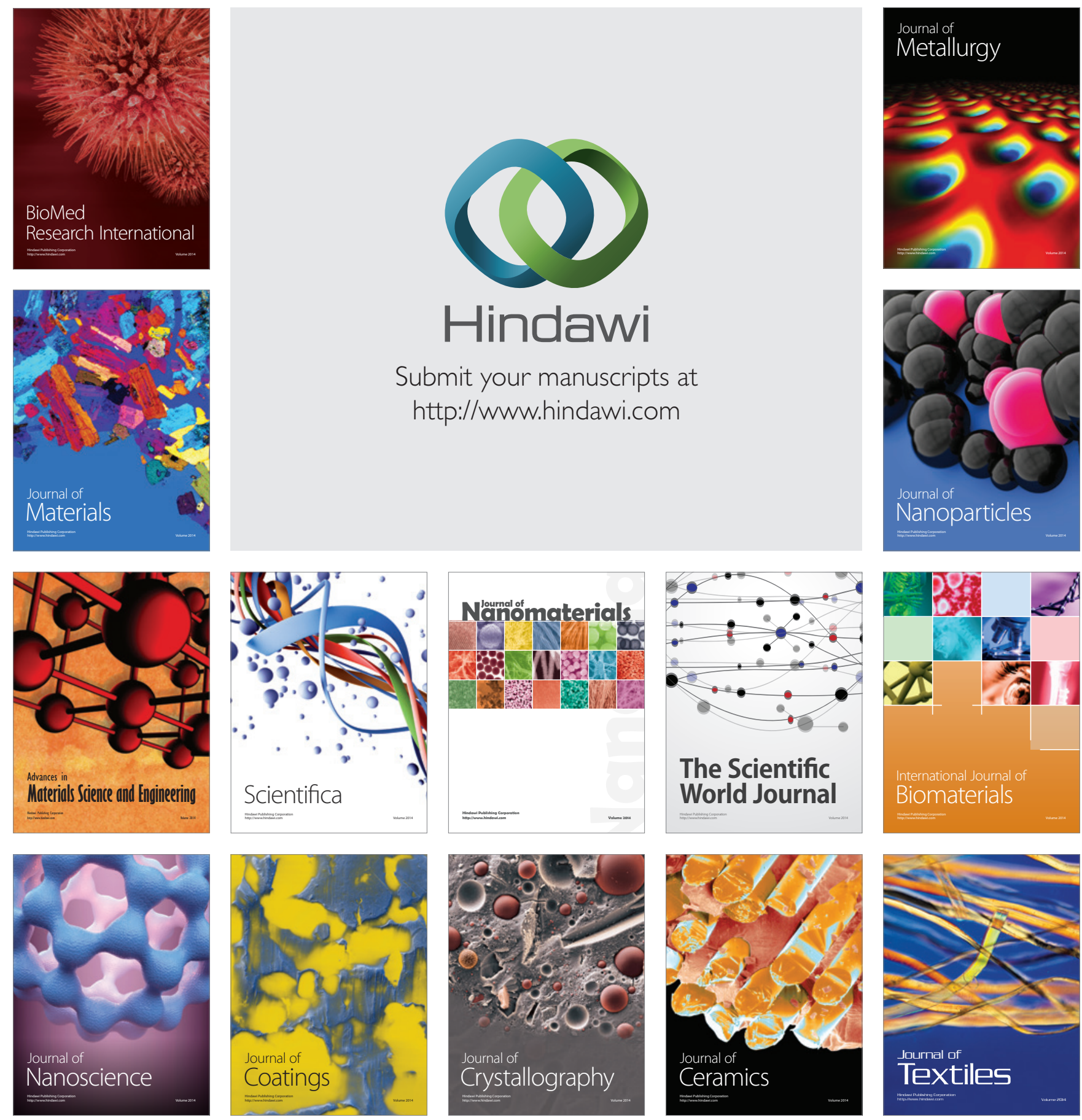